\title{
Patients' Data Security in Telemedicine Consultation in a "New Normal" Post COVID-19 Perspective
}

\author{
${ }^{1}$ Joydeep Dey, ${ }^{2}$ Dr Bappaditya Chowdhury, ${ }^{3}$ Dr Arindam Sarkar, ${ }^{4}$ Dr Sunil Karforma \\ ${ }^{1}$ State Aided College Teacher \& Head, \\ Department of Computer Science, \\ M.U.C. Women's College, Burdwan \\ ${ }^{2}$ Neuropsychiatrist, \\ Belle Vue Hospital, Kolkata \\ ${ }^{3}$ Assistant Professor, \\ Department of Computer Science \& Electronics, \\ R.K.M. Vidyamandira, Belur Math, Howrah \\ ${ }^{4}$ Professor \& Head, \\ Department of Computer Science, \\ The University of Burdwan \\ Corresponding Author Email: joydeepmcabu@gmail.com
}

\begin{abstract}
:
Corona virus has challenged the global health through its asymptomatic carrier. An emergent cameo has been seen towards telepsychiatric for the COVID19 patients and related members. Plenty of psychiatric complications were observed in such patients. Mental treatments are essential at par with the COVID-19 treatments. The frontline COVID-19 warriors like physicians, nurses, hospital staffs, hospital drivers, etc are at greater risks of being infected by the coronavirus and mental challenges. A telepsychiatric way to ensure proper psychiatric treatment has been magnified in the context of post-COVID "New Normal". We have to learn to dwell safely with the said virus. The psychiatric complications are anxiety, hopelessness, acute depression, fear, etc. If such mental illness being ignored then it leads severe catastrophic disruption. A secured mechanism should be deployed in telemedicine consultations with respect to the patients' and doctors' confidential data. Such data should not be shared without encryption over the public medical networks. This article discusses the relevance of data security in the COVID-19 telepsychiatry, and the remote patients are safely treated globally from their isolates without any physical contacts.
\end{abstract}

Keywords: Coronavirus, Telepsychiatry, Patients' Data Security 


\section{INTRODUCTION:}

Telepsychiatry is a combination of psychiatrists and internet friendly computerized system which deals remote patients, which fits best at the current scenario of home-staying [1]. Deployment of telepsychiatry can reduce the COVID-19 transmission rate. The post-COVID-19 has created a new dimension on the larger normalcy perspectives. Telepsychiatry acts as interface beween the patients and psychiatrists, and enabling them to have consultations without any physical hospital visits. Thus, they are safe-guard against the COVID-19 attacks too. Moreover, travelling costs, nascomial infections, body wear and tear, physical contamination with infected patients, allied medical costs, were made to zero in such telepsychiatry consultations. It would help the socioeconomic status of the people too.

Pfefferbaum B. et al. had stated the mental sickness to the Covid-19 health care professionals. A collective mental support and psychiatric monitoring are indeed needed to fight out the pandemic [2]. King J. S. had explained the need of healthcare reforms in the light of COVID19 [3].The most important feature of any telepsychiatry is to protect the patients' data from the intruders. Intruders are ready to steal and manipulate those medical data during the network transmission. Such attack is commonly termed as the Man-In-The-Middle attack. Furthermore, strong authentication protocol is a compulsory organ of telepsychiatry systems. A telepsychiatry system is considered to be stronger when the intruders are unable to diffuse the patients' data within the session. Any of the classical encryption algorithms is used, but the secret key is kept secret between the patients and psychiatrists.

\section{MEDICAL DATA SECURITY ASPECTS:}

Another security aspect in telepsychiatry is the key exchange problem between the patients and psychiatrists. Under any symmetric key communication, there is a secret key exchange between these two users of the system. While exchanging, intruders can easily seize that secret key to manipulate the entire Telepsychiatry consultation session. Moreover, the telepsychiatry credentials of the patients' and psychiatrists' are under vulnerable threats due to users' compromization. While performing payment transactions here, the patients' payment credentials may be leaked. Intruders can steal their banking information. So patients are advised to opt for secure digital wallet option with having facility of end-to-end encryption. Most importantly, the conversations between patients and psychiatrists are mandatorily to be confined. It is the most vulnerable conduct of data breach in such online consultations. Once such confidential data gets compromised, it affects the insurance claims, and a negative societal impact. Intruding on those conversations can lead to severe mental loss to the patients. 


\section{SOLUTION EFFECTS:}

Telepsychiatry has helped the mental states of those COVID patients and allied members. It is a learning curve for us this era of post-COVID "New Normal". Mental care is to be done parallel for treating COVID patients from their homes or COVID hospitals. By integrating behavioural health care and no physical visits to the hospitals, has raised the performances of the telepsychiatry systems. Nguyen H. C. et al. [4] have shown in their study that COVID suspected people are more depressed and found to be with low quality of life. Hence, the telepsychiatry is doing well by reducing the burden of the hospitals and breaking the COVID transmission chain. Regular follow-ups and with no delay is possible through such online telepsychiatry. Transportation and stigmatization barriers do not affect the telepsychiatry functioning. Patients are comfortable in telling / discussing their psychiatric complications in a homely environment through online bidirectional communication. It involves no travelling costs and body wear and tear for the COVID patients.

\section{NEWER APPROACHES IN HEALTH CARE:}

Hospitals were not prepared for such unprecedented pandemic due to coronavirus. But they are adopting with emerging technologies on digital platforms to provide health care services to remote patients. To emphasize more on mental states, they are persuading more on telepsychiatry systems. With more grappling capacity with COVID, there is a spike in number of hospitals adopting for sustainable developments. Patients will be getting more flexibility from such adaptation. It may be stated as a positive thing that hospitals are more proactive towards shifting another way of treatment in terms of online health care. High speed servers with broadband facilities are needed to run such online telepsychiatry systems. Hospitals are motivating their patients to opt for online services, unless an emergency not happened. Post-COVID-19 Telepsychiatry supports a lot towards healing the mental states of the COVID patients and other allied patients. Currently, most of such developed Telepsychiatry systems works on end-to-end encryption having a terminal delivery of the data packets to the destination application ports. Still there are no such system available having full proof securities. A strong Telepsychiatry system depends on its robustness in terms of patients' and psychiatrists' medical data confidentiality.

\section{CONCLUSION:}

When an unprecedented pandemic strikes locally then its rippling effects affect the entire community of the people. This is a matter of compassion and support to all the affected COVID19 patients and their allied persons including the health care givers. To deal with novel corona virus, we need to strive ahead. In this context of post-COVID "New Normal", with the emergence of digital infrastructure, telepsychiatry support has been functioning in a strengthen manner. Psychiatric issues that arise while isolation or quarantined days can be reduced through mental care of telepsychiatry. COVID-19 positive patients can access online telepsychiatry 
services to reduce their mental pressures and dwell in good mentality. Moreover, treating physicians, health care staffs, frontline warriors, COVID19 patients' allied members, COVID19 suspects, etc can also take the opportunity of telepsychiatry services to treat their mental sickness in such critical post-COVID-19 "New Normal" era. Loads of researches are in progress to develop secured telemedicine consultation systems, where patients and psychiatrists will generate their secret keys without any actual key exchange. Neural cryptography is an excellent technique to serve this security challenge. Furthermore, it can be procured efficiently with Telepsychiatry systems with efficacy. The key challenge is for the under-privileged patients having no or less computer literacy.

\section{ACKNOWLEDGEMENT:}

Authors acknowledge the moral and congenial environment given at Maharajadhiraj Uday Chand Women's College, Burdwan, West Bengal, India.

\section{REFERENCES:}

1. Mehrotra A, Ray K, Brockmeyer D M, Barnett M L, Bender J A. Rapidly Converting to "Virtual Practices": Outpatient Care in the Era of Covid-19. The New England Journal of Medicine 2020; DOI: 10.1056/CAT.20.0091

2. Pfefferbaum B. North CS. Mental Health and the Covid-19 Pandemic. The New England Journal of Medicine 2020; DOI: 10.1056/NEJMp2008017.

3. King J S. Covid-19 and the Need for Health Care Reform. The New England Journal of Medicine 2020; DOI: 10.1056/NEJMp2000821.

4. Nguyen, H. C., Nguyen, M. H., Do, B. N., Tran, C. Q., Nguyen, T., Pham, K. M., Pham, L. V., Tran, K. V., Duong, T. T., Tran, T. V., Duong, T. H., Nguyen, T. T., Nguyen, Q. H., Hoang, T. M., Nguyen, K. T., Pham, T., Yang, S. H., Chao, J. C., and Duong, T. V, People with Suspected COVID-19 Symptoms Were More Likely Depressed and Had Lower Health-Related Quality of Life: The Potential Benefit of Health Literacy. Journal of clinical medicine, 9(4);E965. https://doi.org/10.3390/jcm9040965, 2020. 